\title{
THE DENSITY OF ALTERNATION POINTS IN RATIONAL APPROXIMATION
}

\author{
P. B. BORWEIN, ${ }^{*}$ A. KROÓ, ${ }^{*}$ R. GROTHMANN ${ }^{*}$ AND E. B. SAFF ${ }^{* *}$
}

(Communicated by Irwin Kra)

\begin{abstract}
We investigate the behavior of the equioscillation (alternation) points for the error in best uniform rational approximation on $[-1,1]$. In the context of the Walsh table (in which the best rational approximant with numerator degree $\leq m$, denominator degree $\leq n$, is displayed in the $n$th row and the $m$ th column), we show that these points are dense in $[-1,1]$, if one goes down the table along a ray above the main diagonal $(n=[\mathrm{cm}], c<1)$. A counterexample is provided showing that this may not be true for a subdiagonal of the table. In addition, a Kadec-type result on the distribution of the equioscillation points is obtained for asymptotically horizontal paths in the Walsh table.
\end{abstract}

\section{StATEMent OF Results}

Denote by $\mathscr{R}_{m, n}$ the rational functions with numerator in $\Pi_{m}$, the set of algebraic real polynomials of degree at most $m$, and denominator in $\Pi_{n}$. Then the best approximation $r_{m, n}^{*}=p_{m, n}^{*} / q_{m, n}^{*}$ in $\mathscr{R}_{m, n}$ to $f \in C[-1,1]$ with respect to the uniform norm

$$
\|g\|_{[-1,1]}:=\sup \{|g(x)|: x \in[-1,1]\}
$$

is unique and is characterized by an equioscillation property [M], i.e., there are $m+n+2-d$ points

$$
-1 \leq x_{1}^{(m, n)}<\cdots<x_{m+n+2-d}^{(m, n)} \leq 1,
$$

where

$$
d:=d(m, n):=\min \left\{m-\operatorname{deg} p_{m, n}^{*}, n-\operatorname{deg} q_{m, n}^{*}\right\},
$$

such that for a $\sigma= \pm 1$ and all $k=1, \ldots, m+n+2-d$

$$
f\left(x_{k}^{(m, n)}\right)-r_{m, n}^{*}\left(x_{k}^{(m, n)}\right)=\sigma(-1)^{k}\left\|f-r_{m, n}^{*}\right\|_{[-1,1]} .
$$

Received by the editors June 9, 1987 and, in revised form, March 28, 1988.

1980 Mathematics Subject Classification (1985 Revision). Primary 41A20.

Key words and phrases. Rational approximation, extreme points, best approximants.

* The research of these authors was done while visiting the Institute for Constructive Mathematics, University of South Florida, Tampa.

** The research of this author was supported, in part, by the National Science Foundation under grant DMS-8620098. 
(Here and below, we assume that $p_{m, n}^{*}$ and $q_{m, n}^{*}$ do not have a common factor.)

Much is known about the behavior of alternation points for best polynomial approximation $(n=0)$. For this case, Lorentz [L] and Kroó and Saff [KS] give examples showing that for a subsequence $\left\{m_{k}\right\}$ the alternation points (1.2), with $m=m_{k}, n=0$, may avoid a subinterval of $[-1,1]$. However, Kadec $[\mathrm{K}]$ proved that there is always a subsequence such that the alternation points behave like the extremal points of the Chebyshev polynomial of degree $m+1$, that is, like $\{\cos [k \pi /(m+1)]\}_{k=0}^{m+1}$. For polynomial approximation, this implies the denseness of the alternation points in $[-1,1]$.

For rational approximation, given $m$ and $n$, we pick any alternation set (1.2) and write

$$
\rho_{m, n}(f):=\sup _{x \in[-1,1]} \min _{k}\left|x-x_{k}^{(m, n)}\right|
$$

as a measure for the density of the alternation set in $[-1,1]$. We shall prove Theorem 1.1. Let $n=n(m)$ satisfy

$$
n(m) \leq n(m+1) \leq n(m)+1, \quad n(m) \leq m,
$$

for $m=0,1, \ldots$ If $f \in C[-1,1], f \notin \mathscr{R}_{m, n(m)}, m=0,1, \ldots$, then

$$
\liminf _{m \rightarrow \infty}\left(\frac{m-n(m)}{\log m}\right) \rho_{m, n(m)}(f)<\infty .
$$

The proof of Theorem 1.1 will be given in $\S 2$.

Remark. Theorem 1.1 applies in the case $n(m)=[\mathrm{cm}]$ for any constant $c \leq 1$, where $[\cdot]$ denotes the greatest integer function. If $c<1$, we deduce from (1.7) that

$$
\liminf _{m \rightarrow \infty} \rho_{m,[\mathrm{~cm}]}(f)=0,
$$

which implies that the alternation points are dense in $[-1,1]$ for such a "ray sequence" of best approximants. On the other hand, we show in Theorem 1.3 below that this density may not hold when $m / n(m) \rightarrow 1$.

Our second result is similar to Kadec's result $[\mathrm{K}]$ on polynomial approximation. We write for $-1 \leq \alpha<\beta \leq 1$ (with $x_{k}^{(m, n)}$ as in (1.2))

$$
N_{m, n}(\alpha, \beta):=\#\left\{x_{k}^{(m, n)}: \alpha \leq x_{k}^{(m, n)} \leq \beta, k=1, \ldots, m+n+2-d\right\} .
$$

Theorem 1.2. Assume, in addition to the hypotheses of Theorem 1.1, that

$$
\lim _{m \rightarrow \infty} \frac{n(m)}{m}=0 \text {. }
$$

Then there exists a subsequence $\Omega$ of $\mathbb{N}$ such that for all $[\alpha, \beta] \subseteq[-1,1]$,

$$
\lim _{\substack{m \rightarrow \infty \\ m \in \Omega}} \frac{N_{m, n(m)}(\alpha, \beta)}{N_{m, n(m)}(-1,1)}=\frac{\arccos \alpha-\arccos \beta}{\pi} .
$$

Finally, we give a counterexample, which shows that Theorems 1.1 and 1.2 cannot be proved for a subdiagonal of the Walsh table. Indeed, for approximation in $\mathscr{R}_{n-1, n}$ it is possible that, for all $n$, the extremal points all reside in an arbitrarily small interval. 
Theorem 1.3. For every $2>\varepsilon>0$, there is a function $f \in C[-1,1]$ such that for each $n=1,2, \ldots$ the error $f-r_{n-1, n}^{*}(f)$ has no alternation points in $(-1+\varepsilon, 1]$.

The proof of Theorem 1.3 will be given in $\S 3$. The results of this paper should be compared with those of Kroó and Peherstorfer [KP] for $L_{1}$-approximation.

\section{Proofs of Theorems 1.1 AND 1.2}

We need the following lemma, which follows easily from classical results. We include the proof for the sake of completeness.

Lemma 2.1. Given $-1 \leq \alpha<\beta \leq 1$ and $n \in \mathbb{N}$ there exists $a p_{n} \in \Pi_{n}$ with

$$
\left\|p_{n}\right\|_{[-1, \alpha] \cup[\beta, 1]}<1 \text {, }
$$

and

$$
\left\|p_{n}\right\|_{[\alpha, \beta]}>c_{1} e^{c_{2} n(\beta-\alpha)},
$$

where $c_{1}, c_{2}>0$ are constants independent of $\alpha, \beta$ and $n$.

In (2.1) and (2.2) the norms are again the sup norms over the indicated set. Proof. Let

$$
T_{m}(x):=\cos (m \arccos x)
$$

denote the Chebyshev polynomial of degree $m$. For $m:=[n / 2], \tau:=$ $(\beta-\alpha) / 2$, set

$$
q_{n}(x):=\frac{1}{2} T_{m}\left(1+\frac{\tau^{2}}{2}-\left(2+\frac{\tau^{2}}{2}\right) \frac{x^{2}}{4}\right) .
$$

Since $\tau \leq 1$ and

$$
T_{m}(1+\eta) \geq \frac{1}{2}(1+\sqrt{2 \eta})^{m}, \quad \eta>0,
$$

we have for some constants $c_{1}, c_{2}>0$ :

$$
q_{n}(0)=\frac{1}{2} T_{m}\left(1+\frac{\tau^{2}}{2}\right) \geq \frac{1}{4}(1+\tau)^{m}>c_{1} e^{n c_{2}(\beta-\alpha)} .
$$

For $x \in[-2,-\tau] \cup[\tau, 2]$,

$$
-1 \leq 1+\frac{\tau^{2}}{2}-\left(2+\frac{\tau^{2}}{2}\right) \frac{x^{4}}{4}<1 .
$$

The lemma now follows with $p_{n}(x):=q_{n}(x-(\alpha+\beta) / 2)$.

Proof of Theorem 1.1. Set $E_{m}(f):=\left\|f-r_{m, n(m)}^{*}\right\|_{[-1,1]}$ for $m \in \mathbb{N}$. Since $f \notin \mathscr{R}_{m, n(m)}$, we have $E_{m}(f)>0$ for all $m \in N$. Also, from (1.6), it follows that $E_{m}(f) \downarrow 0$, and so from elementary theorems about series (cf. [K])

$$
\sum_{m=0}^{\infty} \frac{E_{m}(f)-E_{m+1}(f)}{E_{m}(f)+E_{m+1}(f)}=\infty .
$$


Thus there is a subsequence $\Omega$ of $\mathbb{N}$ with $E_{m}(f)-E_{m+1}(f) \neq 0$ and

$$
\frac{E_{m}(f)+E_{m+1}(f)}{E_{m}(f)-E_{m+1}(f)}<m^{2}
$$

for all $m \in \Omega$.

For $m \in \Omega$, set

$$
R_{m}:=\frac{1}{E_{m}(f)-E_{m+1}(f)}\left(r_{m, n(m)}^{*}-r_{m+1, n(m+1)}^{*}\right) .
$$

At the alternation points

$$
-1 \leq x_{1}^{(m)}<\cdots<x_{m+n(m)+2-d(m)}^{(m)} \leq 1
$$

of $f-r_{m, n(m)}^{*}$ we have with $\sigma= \pm 1$

$$
\sigma(-1)^{k} R_{m}\left(x_{k}^{(m)}\right) \geq 1, \quad k=1, \ldots, m+n(m)+2-d(m) .
$$

Moreover, from (1.6) and (2.12) it follows that $R_{m}=P_{m} / Q_{m}$ with

$$
\begin{gathered}
\operatorname{deg} P_{m}=m+n(m)+1-d(m), \\
\operatorname{deg} Q_{m} \leq 2 n(m)+1-d(m) .
\end{gathered}
$$

Thus $R_{m}-q$ can have at most $m+n(m)+1-d(m)$ zeros, if $q \in \Pi_{m-n(m)}$. Let $c_{1}, c_{2}$ be as in Lemma 2.1. For $m \in \Omega$, let $x_{m}^{*} \in[-1,1]$ satisfy

$$
\min _{k}\left|x_{m}^{*}-x_{k}^{(m)}\right|=\rho_{m, n(m)}(f)=: t_{m} \text {. }
$$

If $x_{m}^{*} \in\left[-1, x_{1}^{(m)}\right]$, we let $p_{m-n(m)}$ be the polynomial that satisfies Lemma 2.1 with $\alpha=-1$ and $\beta=x_{1}^{(m)}$. From (2.12) and (2.1) it follows that $R_{m} \pm p_{m-n(m)}$ has $m+n(m)+1-d(m)$ zeros in $\left(x_{1}^{(m)}, 1\right]$ and hence is zero-free in $\left[-1, x_{1}^{(m)}\right]$. Thus

$$
c_{1} e^{c_{2} t_{m}(m-n(m))} \leq\left\|R_{m}\right\|_{[-1,1]}<m^{2},
$$

where the last inequality follows from (2.9). If $x_{m}^{*} \in\left[x_{m+n(m)+2-d(m)}^{(m)}, 1\right]$, we use Lemma 2.1 with $\alpha=x_{m+n(m)+2-d(m)}^{(m)}$ and $\beta=1$ and again we get (2.16). Otherwise denote the zeros of $R_{m}$ by $y_{k}^{(m)}$, where

$$
x_{1}^{(m)}<y_{1}^{(m)}<x_{2}^{(m)}<\cdots<y_{m+n(m)+1-d(m)}^{(m)}<x_{m+n(m)+2-d(m)}^{(m)},
$$

and set $y_{0}^{(m)}:=x_{1}^{(m)}, y_{m+n(m)+2-d(m)}^{(m)}:=x_{m+n(m)+2-d(m)}^{(m)}$. Then $\left|y_{k}^{(m)}-y_{k+1}^{(m)}\right| \geq t_{m}$ for some $k=k^{*}$. As above, counting the zeros of $R_{m} \pm\left(p_{m-n(m)}-1\right) / 2$, where $p_{m-n(m)}$ satisfies Lemma 2.1 with $\alpha=y_{k^{*}}^{(m)}$ and $\beta=y_{k^{*}+1}^{(m)}$, yields

$$
\frac{1}{2}\left(c_{1} e^{c_{2} t_{m}(m-n(m))}-1\right)<m^{2} .
$$


By (2.16) or (2.18) we get for a constant $c_{3}>0$

$$
t_{m}(m-n(m)) \leq c_{3} \log m,
$$

which yields (1.7).

Proof of Theorem 1.2. It suffices to prove (1.11) for the case $\alpha=-1$. In fact, it is enough to show that

$$
\limsup _{\substack{m \rightarrow \infty \\ m \in \Omega}} \frac{N_{m, n(m)}(-1, \beta)}{N_{m, n(m)}(-1,1)} \leq \frac{\pi-\arccos \beta}{\pi},
$$

since replacing $x$ by $-x$ and $\beta$ by $-\beta$, we get the corresponding lower estimate for lim inf. Let $m-n(m)=s(m)+l(m)$, where $s(m)$ is to be determined later. With the notations of the previous proof, set for $m \in \Omega$,

$$
\begin{gathered}
q_{m}(x):=\frac{1}{2} T_{s(m)}(x+1-\beta) T_{l(m)}(x), \\
N(m):=\#\left\{x \in(\beta, 1]:\left|T_{l(m)}(x)\right|=1,\left|q_{m}(x)\right|>m^{2}\right\},
\end{gathered}
$$

where $T_{k}$ denotes the $k$ th the Chebyshev polynomial, $\left\|T_{k}\right\|_{[-1,1]}=1$. Then $R_{m}-q_{m}$ has at least $N(m)-1$ zeros in $(\beta, 1]$. Thus it can have at most $m+n(m)+2-d(m)-N(m)$ zeros in $[-1, \beta]$. Hence

(2.23) $\limsup _{m \rightarrow \infty} \frac{N_{m, n(m)}(-1, \beta)}{N_{m, n(m)}(-1,1)} \leq \limsup _{m \rightarrow \infty} \frac{m+n(m)+2-d(m)-N(m)}{m+n(m)+2-d(m)}$

$$
=1-\liminf _{m \rightarrow \infty} \frac{N(m)}{m},
$$

since between two alternation points of $R_{m}$ in $[-1, \beta]$ is one zero of $R_{m}-q_{m}$ and since $n(m) / m \rightarrow 0(d(m) \leq n(m))$. In (2.23) and the rest of the proof, all limits are for $m \in \Omega$. Now choose $s(m)$ such that

$$
\lim _{m \rightarrow \infty} \frac{s(m)}{\log m}=\infty, \quad \lim _{m \rightarrow \infty} \frac{l(m)}{m}=1 .
$$

Then the first equation in (2.24) together with (2.5) yields

$$
\lim _{m \rightarrow \infty}\left(\inf \left\{x \in(\beta, 1]: \frac{1}{2} T_{s(m)}(x+1-\beta)>m^{2}\right\}\right)=\beta .
$$

Also, for $\beta<\widetilde{\beta}<1$, it follows from the second equation in (2.24) that

$$
\lim _{m \rightarrow \infty} \frac{\#\left\{x \in(\tilde{\beta}, 1]:\left|T_{l(m)}(x)\right|=1\right\}}{m}=\frac{\arccos \tilde{\beta}}{\pi} .
$$

Finally, (2.25) and (2.26) yield (with (2.21) and (2.22))

$$
\liminf _{m \rightarrow \infty} \frac{N(m)}{m} \geq \frac{\arccos \beta}{\pi},
$$

which together with (2.23) gives (2.20). 


\section{Proof of Theorem 1.3}

For $\alpha_{1} \leq \cdots \leq \alpha_{n}<0$ we denote by $V_{n-1}\left(\alpha_{1}, \ldots, \alpha_{n}\right)=x^{n-1}+\cdots$ the monic polynomial that minimizes

$$
\left\|\frac{p_{n-1}(x)}{\prod_{k=1}^{n}\left(x-\alpha_{k}\right)}\right\|_{[0,1]}
$$

among all monic polynomials $p_{n-1}$ of degree $n-1$. Set

$$
r_{n-1}\left(\alpha_{1}, \ldots, \alpha_{n}\right)(x):=\frac{V_{n-1}\left(\alpha_{1}, \ldots, \alpha_{n}\right)(x)}{\prod_{k=1}^{n}\left(x-\alpha_{k}\right)} .
$$

Then from the Haar condition (cf. [M, $\S 3.2]), r_{n-1}\left(\alpha_{1}, \ldots, \alpha_{n}\right)$ is uniquely determined and equioscillates $n$ times in $[0,1]$. Moreover, these $n$ equioscillation points are the only extremal points of $r_{n-1}\left(\alpha_{1}, \ldots, \alpha_{n}\right)$ in $[0,1]$, since $r_{n-1}\left(\alpha_{1}, \ldots, \alpha_{n}\right)-c$ can have at most $n$ zeros for each $c \in \mathbf{R}$ and since $r_{n-1}\left(\alpha_{1}, \ldots, \alpha_{n}\right)$ has all its $n-1$ zeros in $[0,1]$. Also, zero must be one of the equioscillation points, since $\left|r_{n-1}\left(\alpha_{1}, \ldots, \alpha_{n}\right)(x)\right|$ decreases between $\alpha_{n}$ and the first zero of $r_{n-1}\left(\alpha_{1}, \ldots, \alpha_{n}\right)$.

We need the following lemmas.

Lemma 3.1. Let $\alpha_{1} \leq \cdots \leq \alpha_{n}<0$ and $\beta_{1} \leq \cdots \leq \beta_{n}<0$ satisfy $\alpha_{k} \leq$ $\beta_{k}$ for $k=1, \ldots, n$. Then the equioscillation points $x_{1}<\cdots<x_{n}$ of $r_{n-1}\left(\alpha_{1}, \ldots, \alpha_{n}\right)$ and $y_{1}<\cdots<y_{n}$ of $r_{n-1}\left(\beta_{1}, \ldots, \beta_{n}\right)$ satisfy $x_{k} \geq y_{k}$ for $k=1, \ldots, n$.

Proof. It suffices to prove the lemma in the case $\alpha_{k}=\beta_{k}$ for $k \neq k^{*}, \alpha_{k^{*}}<$ $\beta_{k^{*}}$, since we can transfer $\alpha_{n}$ to $\beta_{n}, \ldots, \alpha_{1}$ to $\beta_{1}$ successively. Define

$$
\begin{aligned}
& C_{\alpha}:=1 /\left\|r_{n-1}(\underline{\alpha})\right\|_{[0,1]}, \\
& C_{\beta}:=1 /\left\|r_{n-1}(\underline{\beta})\right\|_{[0,1]},
\end{aligned}
$$

where $\underline{\alpha}=\left(\alpha_{1}, \ldots, \alpha_{n}\right), \underline{\beta}=\left(\beta_{1}, \ldots, \beta_{n}\right)$, and set

$$
\begin{aligned}
q(x) & :=C_{\alpha}\left(x-\beta_{k^{*}}\right) V_{n-1}(\underline{\alpha})(x)-C_{\beta}\left(x-\alpha_{k^{*}}\right) V_{n-1}(\underline{\beta})(x) \\
& =\left(C_{\alpha} r_{n-1}(\underline{\alpha})(x)-C_{\beta} r_{n-1}(\underline{\beta})(x)\right)\left(x-\alpha_{k^{*}}\right)\left(x-\beta_{k^{*}}\right) \prod_{k \neq k^{*}}\left(x-\alpha_{k}\right) .
\end{aligned}
$$

By the equioscillation, we have $\left(y_{1}=0\right)$

$$
q\left(y_{n}\right) \leq 0, q\left(y_{n-1}\right) \geq 0, \ldots, q(0)=0 .
$$

It is easy to see that $C_{\alpha}>C_{\beta}$. Thus there is a point $x>y_{n}$ with $q(x)>0$. For the (necessarily real) zeros $\xi_{1} \leq \cdots \leq \xi_{n}$ of $q$ (some zeros may be counted twice), this implies $\xi_{k} \geq y_{k}$ for $k=1, \ldots, n$. We also have $\left(x_{1}=0\right)$

$$
q\left(x_{n}\right) \geq 0, q\left(x_{n-1}\right) \leq 0, \ldots, q(0)=0 .
$$


For every $\varepsilon>0$, there is a polynomial $\tilde{q} \in \Pi_{n}$ with highest coefficient $C_{\alpha}-C_{\beta}$ and real zeros $\tilde{\xi}_{1} \leq \cdots \leq \tilde{\xi}_{n}$ such that

$$
\begin{gathered}
\tilde{q}\left(x_{n}\right)>0, \tilde{q}\left(x_{n-1}\right)<0, \ldots, \tilde{q}(0)<0 \quad \text { for } n \text { even }, \\
\tilde{q}(0)>0 \quad \text { for } n \text { odd }, \\
\left|\widetilde{\xi}_{k}-\xi_{k}\right|<\varepsilon, \quad k=1, \ldots, n .
\end{gathered}
$$

It follows that $\tilde{\xi}_{1}<0$ and thus $x_{k}>\widetilde{\xi}_{k}$ for $k=1, \ldots, n$. Since $\varepsilon>0$ is arbitrary, this implies $x_{k} \geq \xi_{k} \geq y_{k}$ for $k=1, \ldots, n$.

Lemma 3.2. Given $0<\varepsilon<1$ there is an increasing sequence $a_{1}<a_{2}<\cdots<0$, such that $r_{n-1}\left(a_{1}, \ldots, a_{n}\right)$ has no extremal points in $(\varepsilon, 1]$ for $n \geq 1$.

Proof. Set $a_{1}:=-\varepsilon / 4$. We will construct $a_{n}$ "by induction such that the function

$$
f_{n}(x):=\frac{\prod_{k=2}^{n}\left(x+2 a_{k}\right)}{\prod_{k=1}^{n}\left(x-a_{k}\right)}
$$

alternates in sign in the points

$$
0=\delta_{1, n}<\cdots<\delta_{n, n}<\varepsilon
$$

and satisfies

$$
\left|f_{n}\left(\delta_{k, n}\right)\right|>\left|f_{n}(x)\right| \quad \text { for } k=1, \ldots, n, x \in[\varepsilon, 1] .
$$

If we have this sequence, $r_{n-1}\left(a_{1}, \ldots, a_{n}\right)$ cannot have an alternation point in $(\varepsilon, 1]$ for $n \geq 2$, since otherwise for a suitably chosen $\gamma \in \mathbf{R}$ the function $f_{n}-\gamma r_{n-1}\left(a_{1}, \ldots, a_{n}\right)$ has a zero in each interval $\left(\delta_{k, n}, \delta_{k+1, n}\right)$ and an additional zero in $\left(\delta_{n, n}, 1\right)$.

We observe now that $f_{1}(x)=1 /\left(x-a_{1}\right)$ is decreasing in $[0,1]$ and satisfies (3.12) with $\delta_{1,1}=0$. Having constructed $a_{1}, \ldots, a_{n-1}$, we observe that

$$
\lim _{a_{n} \rightarrow 0^{-}} \frac{x+2 a_{n}}{x-a_{n}}=1 \text { uniformly on }[\lambda, 1]
$$

for all $\lambda>0$. Thus, for $\left|a_{n}\right|$ sufficiently small, (3.12) will be satisfied for $\delta_{k, n+1}:=\delta_{k-1, n}, k=3, \ldots, n+1$ and for $\delta_{1, n+1}:=\delta_{1, n}=0$. Thus it remains to show the existence of $\delta_{2, n+1}$. Since $f_{n+1}(0)=-2 f_{n}(0)$, this follows from (3.13) by choosing $\left|a_{n}\right|$ small enough.

Proof of Theorem 1.3. We will prove the theorem on the interval $[0,1]$. Choose $a_{1}, a_{2}, \ldots$ as in Lemma 3.2 with $\varepsilon / 2$ replacing $\varepsilon$. Let, for $b_{k}>0$,

$$
S_{n}(x):=\sum_{k=1}^{n} \frac{b_{k}}{x-a_{2 k}} .
$$

We now use a result in [B] stating that the best approximation to $S_{n}$ out of $\mathscr{R}_{n-2, n-1}$ has the form

$$
r_{n-2, n-1}^{*}\left(S_{n}\right)(x)=\frac{p_{n-2}^{*}(x)}{\prod_{k=1}^{n-1}\left(x-a_{2 k+1}^{*}\right)},
$$


where $p_{n-2}^{*}$ is of degree $n-2$ and

$$
a_{2}<a_{3}^{*}<a_{4}<\cdots<a_{2 n-1}^{*}<a_{2 n}<0 .
$$

Thus, by the equioscillation property (1.4), there is a constant $c_{n}$ such that

$$
S_{n}-r_{n-2, n-1}^{*}\left(S_{n}\right)=c_{n} r_{2 n-2}\left(a_{2}, a_{3}^{*}, a_{4}, \ldots, a_{2 n}\right) \text {. }
$$

Since $r_{2 n-2}\left(a_{1}, \ldots, a_{2 n-1}\right)$ has no alternation point in $(\varepsilon / 2,1]$, Lemma 3.1 shows that $S_{n}-r_{n-2, n-1}^{*}\left(S_{n}\right)$ has no alternation point in $(\varepsilon / 2,1]$. We choose the $b_{k}$ 's such that

$$
\text { the series } f(x)=\sum_{k=1}^{\infty} \frac{b_{k}}{x-a_{2 k}} \text { converges uniformly on }[0,1] \text {, }
$$

and

$$
\begin{aligned}
& r_{n-2, n-1}^{*}(f) \text { is close enough to } r_{n-2, n-1}^{*}\left(S_{n}\right) \text { to guarantee that } \\
& f-r_{n-2, n-1}^{*}(f) \text { has no alternation point in }(\varepsilon, 1] .
\end{aligned}
$$

For (3.19) we used the fact (cf. [W]) that the best approximation operator is continuous in $S_{n}$, since $r_{n-2, n-1}^{*}\left(S_{n}\right)$ is nondegenerate (i.e. $d=0$ in (1.3)).

\section{REFERENCES}

[B] M. Barnsley, The bounding properties of the multipoint Pade approximant to a series of Stieltjes on the real line, J. Math. Phys. 14 (1973), 299-313.

[K] M. I. Kadec, On the distribution of points of maximal deviation in the approximation of continuous functions by polynomials, Uspekhi Mat. Nauk. 15 (1960), 199-202.

[KP] A. Kroó and F. Peherstorfer, Interpolating properties of best rational $L_{1}$-approximations, Constr. Approx. 4 (1988), 97-106.

[KS] A. Kroó and E. B. Saff, The density of extreme points in complex polynomial approximation, Proc. Amer. Math. Soc. 103 (1988), 203-209.

[L] G. G. Lorentz, Distribution of alternation points in uniform polynomial approximation, Proc. Amer. Math. Soc. 92 (1984), 401-403.

[M] G. Meinardus, Approximation theory: Theory and numerical methods, Springer tracts in natural philosphy, Vol. 13, Springer-Verlag, New York, 1967.

[W] H. Werner, On the rational Tschebyscheff operator, Math. Z. 86 (1964), 317-326.

Department of Mathematics, Dalhousie University, Halifax, Nova Scotia, Canada B3H $4 \mathrm{H} 8$

Current address (A. Kroó): Mathematical Institute of the Hungarian Academy of SCIENCES, Budapest, Reáltanoda U. 13-15, H-1053 Hungary

Current address (R. GrothmanN) : Mathem. Geogr. Fakultat, Katholische Universität Eichstätt, OStenstrasse 18, D-8078 Eichstätt, West Germany

Current address (E. B. SAFF) : Institute for Constructive Mathematics, Department of Mathematics, University of South Florida, Tampa, Florida 33620 USA 